\title{
Implications of NQO1 in cancer therapy
}

\author{
Eun-Taex $\mathrm{Oh}^{1,2} \mathcal{E}$ Heon Joo Park ${ }^{2,3, *}$ \\ ${ }^{1}$ Department of Biomedical Sciences, ${ }^{2}$ Hypoxia-related Disease Research Center, and ${ }^{3}$ Department of Microbiology, School of Medicine, \\ Inha University, Incheon 22212, Korea
}

\begin{abstract}
$\mathrm{NAD}(\mathrm{P}) \mathrm{H}:$ quinone oxidoreductase (NQO1), an obligatory two-electron reductase, is a ubiquitous cytosolic enzyme that catalyzes the reduction of quinone substrates. The NQO1mediated two-electron reduction of quinones can be either chemoprotection/detoxification or a chemotherapeutic response, depending on the target quinones. When toxic quinones are reduced by NQO1, they are conjugated with glutathione or glucuronic acid and excreted from the cells. Based on this protective effect of NQO1, the use of dietary compounds to induce the expression of NQO1 has emerged as a promising strategy for cancer prevention. On the other hand, NQO1-mediated two-electron reduction converts certain quinone compounds (such as mitomycin C, E09, RH1 and $\beta$-lapachone) to cytotoxic agents, leading to cell death. It has been known that NQO1 is expressed at high levels in numerous human cancers, including breast, colon, cervix, lung, and pancreas, as compared with normal tissues. This implies that tumors can be preferentially damaged relative to normal tissue by cytotoxic quinone drugs. Importantly, NQO1 has been shown to stabilize many proteins, including p53 and p33ING1b, by inhibiting their proteasomal degradation. This review will summarize the biological roles of NQO1 in cancer, with emphasis on recent findings and the potential of NQO1 as a therapeutic target for the cancer therapy. [BMB Reports 2015; 48(11): 609-617]
\end{abstract}

\section{INTRODUCTION}

NQO1 (NAD(P)H:quinone oxidoreductase 1) is a cytosolic flavoenzyme, which is also known as DT-diaphorase (EC 1.6.99.2) (1). NQO1 is expressed in various tissues, and its gene expression is regulated by the ARE (antioxidant response element), both in normal condition and during oxidative stress conditions (2). The NQO1 gene contains ARE in its promoter region and is regulated by the nuclear factor (erythroid- der-

*Corresponding author. Tel: +82-32-860-9800; Fax: +82-32-8858302; E-mail: park001@inha.ac.kr

http://dx.doi.org/10.5483/BMBRep.2015.48.11.190

Received 20 September 2015

Keywords: NQO1, Cancer prevention, Cancer therapy eved)-like 2 (Nrf2) (3). The NQO1 gene has been shown to be activated together with other Nrf2-induced detoxifying enzyme genes, such as GST (glutathione S-transferase) and HO-1 (heme oxygenase), in response to antioxidants, ionizing radiation, xenobiotics, heat shock, electrophiles, hypoxia, and heavy metals $(1,4)$.

The catalytic enzyme properties of NQO1 were first reported by Ernster and Navazio in 1958 (5). NQO1 is considered as an anticancer enzyme since it protects cells from oxidative stresses through inhibition of quinones from entering the one electron reduction to semiquinone free radicals and ROS (reactive oxygen species) $(6,7)$. Thus, the use of dietary compounds to induce the expression of NQO1 has emerged as a promising strategy for cancer prevention $(8,9)$. Recent studies have revealed that NQO1 activity is related to the risks of lung cancer $(6,7,10,11)$ or cancer of other organs $(12,13)$. Development of several types of human cancers has been shown to be due to NQO1 polymorphisms (14-18). Recent meta-analysis studies have shown that, in the human representative catalytic mutated NOQ1 gene (C609T) located on chromosome region $16 q 22$, replacement of cytosine with thymidine $(609 \mathrm{C}>\mathrm{T})$ express substitution of serine for proline, thereby reducing the NQO1 enzyme activity, leading to development of several types of human cancers $(11,16-21)$.

Although a lowered or absent NQO1 activity has been correlated with increased susceptibility for development of human cancers $(11,21)$, numerous studies found that NQO1 is upregulated in a number of cancers such as breast cancer, pancreatic cancer, colorectal cancer, cholangiocarcinoma, uterine cervical cancer, melanoma, and lung cancer $(22,23)$. In breast, colorectal and cervical cancers, the high-level expression of NQO1 was found to be associated with the late clinical stage of the disease, poor differentiation and lymph node metastasis $(22,23)$. Consistently, breast and cervical cancer patients with high NQO1 expression levels show lower DFS (Disease-free Survival) and 5-year OS (Overall Survival) rates, as compared to patients having lower NQO1 expression $(22,23)$. In addition, NQO1 activity in many cancers is significantly higher than that in adjacent normal tissues (1, 24-26).

With its unique property of transferring two-electron by using either NADH or NADPH as reducing cofactor, NQO1 catalyzes the natural and exogenous quinones and quinineimines into hydroquinones, which are toxic (27-29). Accordingly,

ISSN: 1976-670X (electronic edition)

Copyright (c) 2015 by the The Korean Society for Biochemistry and Molecular Biology

(ㄷ) This is an open-access article distributed under the terms of the Creative Commons Attribution Non-Commercial License (http://creativecommons.org/licenses/by-nc/4.0) which permits unrestricted non-commercial use, distribution, and reproduction in any medium, provided the original work is properly cited. 
there has been considerable effort to develop bioreductive anticancer drugs, such as mitomycin $\mathrm{C}$, E09, RH1, $\beta$-lapachone, and 17AAG, which are activated specifically by NQO1 and, thus are preferentially toxic to cancer cells (30-38). Importantly, it has been reported that ionizing radiation (2-4 Gy) $(1,38-40)$, cisplatin $(1)$, or hyperthermia $\left(41-42^{\circ} \mathrm{C}\right)(41,42)$ increased the NQO1 expression levels in various human and animal cancer cells, and sensitized the cells to $\beta$-lapachone, both in vitro and in vivo.

Recent emerging studies have revealed the protective roles for NQO1 regardless of its enzymatic activities (43). NQO1 structurally binds to the important tumor suppressor p53 and increases its protein stability by inhibiting proteasomal degradation (43). Furthermore, NQO1 appears to regulate the protein stability of other proteins such as p33, p73, p33ING1b, and C/EBP $\alpha$ (44-48).

These studies suggested that NQO1 is a multifunctional antioxidant enzyme and an exceptionally versatile cytoprotector, which contributes to a dual function in tumorigenic progression. This review will describe the biological significances of NQO1 in cancer, with emphasis on recent findings, and the potential of NQO1 as a therapeutic target for cancer therapy.

\section{BIOCHEMICAL PROPERTIES OF NQO1 AND CANCER PREVENTION}

One way to prevent cancer development is suppression of the carcinogenic metabolic activation and preventing the production of ultimate carcinogens (49). Recent studies showed that induction of phase II enzymes, such as GST, HO-1, and NQO1, correlates with inhibition against chemical-mediated tumorigenesis in animal models, during the promotion as well as initiation stages $(8,49)$. Among phase II enzymes, NQO1 has been most extensively studied for its effect in preventing carcinogenesis $(8,50)$. The multiple and general biochemical roles of NQO1 in the protection against the promotion and initiation of cancer, can be summarized into the following four

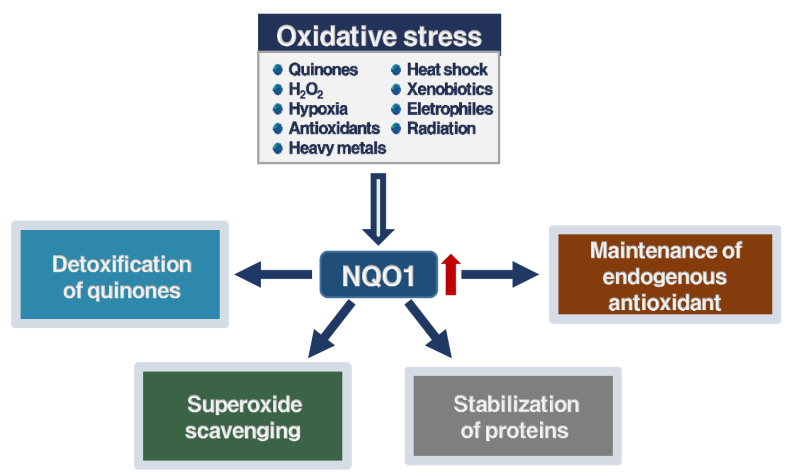

Fig. 1. The biochemical multiple and general roles of NQO1 in protection against the development of cancer. categories (Fig. 1): (i) detoxification of quinone substrates by two-electron reduction; (ii) scavenging of SOD (superoxide anion radicals); (iii) maintenance of the endogenous antioxidants such as ubiquinone and $\alpha$-tocopherol; and (iv) stabilization of the suppressors p53/p73/p33 proteins.

\section{Detoxification of quinones by NQO1}

In various species including rat, zebrafish, mouse, and human, NQO1 is well known as a homodimeric flavoprotein $(2,51$, 52). The best-described and widely accepted function of NQO1 is, as its name suggests, the reduction of quinones (2). Endogenous and environmental quinones are highly reactive molecules that can induce cancers and neurodegenerative diseases (53). NQO1 catalyzes the obligatory single-step twoelectron reduction of the quinones to hydroquinones. Then the hydroquinones can undergo conjugation reactions (e.g., glucuronidation). Consequently, they are readily excreted from the body $(2,50)$. The two-electron reduction activity catalyzed by NQO1 is of benefit to the cell as it prevents generation of free radicals by redox cycle (54). Thus, the detoxification of redox-cycling quinones by NQO1 protects the cells from oxidative stresses and prevents carcinogenesis $(55,49)$. For example, NQO1-mediated reduction of menadione produces its formation of stable hydroquinone to be readily conjugated and excreted from the body (53). This NQO1-mediated two-electron reduction of quinones inhibits the production of unwanted one-electron reduction of them by other enzymes such as cytochrome P450 (50, 56, 57). The oxidoreductase functions have been proposed to involve a hydride transfect between the $\mathrm{NADH}$ and FAD cofactors and from FADH 2 to the quinone substrate $(50,53)$. NQO1 can reduce a very broad range of substrates, including quinones, dichlorophenolindolphenol, quinone-imines, methylene blue, glutathionyl-substituted naphthoquinones, and also azo and nitro compounds $(53,56)$. In addition to the two-electron reduction, NQO1 is also capable of performing four-electron reduction of nitro compounds and azo dyes (58). The ability of NQO1 to reduce the toxicity and carcinogenicity of various quinones has been reviewed comprehensively elsewhere $(59,60)$.

\section{NQO1 as a scavenger of superoxide anion radicals}

Recent studies demonstrated that NQO1 directly scavenges superoxides in an $\mathrm{NAD}(\mathrm{P}) \mathrm{H}$-dependent manner $(27,50)$. This protective effect may be significant for certain tissues, such as vasculature and myocardium, in which NQO1 is highly expressed $(27,61,62)$. In cardiovascular tissues, the high NQO1 enzyme activity compensates for the lows of cardiovascular superoxide dismutase expression in the detoxification reaction of superoxide anion radicals, which are produced by various sources, including xanthine oxidase, $\mathrm{NAD}(\mathrm{P}) \mathrm{H}$ oxidases, mitochondria, and uncoupled NOSs (nitric oxide synthases) in cardiovascular tissues (61). 


\section{NQO1 as an antioxidant enzyme}

There has been known that NQO1 maintains certain endogenous antioxidants in their reduced and active forms (50). Oxidation of $\alpha$-tocopherolquinone, which is produced by vita$\min E$ ( $\alpha$-tocopherol), has antioxidant properties following reduction to $\alpha$-tocopherolhydroquinone (53). NQO1 catalyzes the two-electron reduction of $\alpha$-tocopherolquinone to its hydroquinone form, which then protects against lipid peroxidation of the membranes (61). Furthermore, NQO1 catalyzes the reduction of ubiquinone analogs (coenzyme Q) to their ubiquinol forms in liposomes (53). However, the in vivo role of the above two-electron reduction reactions remains to be elucidated.

\section{NQO1 as a protein stabilizer}

The tumor suppressor p53, which is one of critical transcription factors related to suppression of tumourigenesis, induces either growth arrest or apoptosis, in response to stresses such as DNA damage (43). The p53 protein is regulated via modification and interactions that affect its half-life (44). Under normal conditions, p53 protein is rapidly degraded due to its interaction with $\mathrm{Mdm}-2$ that induces ubiquitination and proteasomal degradation (44). Pro-apoptotic stresses disrupt this interaction between the p53 and Mdm-2 proteins, allowing the p53 to accumulate (63). NQO1 has been shown to stabilize the tumor suppressor p53 protein (43). When NQO1 expression is upregulated in cancer cells under conditions of stress, NQO1 stabilizes p53 by inhibiting its proteasomal degradation. This effect was reversed by the potent NQO1 inhibitor dicoumarol, as well as other inhibitors that compete with $\mathrm{NAD}(\mathrm{P}) \mathrm{H}(43)$. This effect of dicoumarol suggests that it affects a structural change in NQO1, which inhibits interaction between the NQO1 and p53 proteins $(45,46)$. Although the precise mechanism by which NQO1 activity stabilizes p53 is poorly understood, the NQO1-mediated stabilization of p53 represents a unique additional mechanism by which NQO1 may protect against carcinogenesis. In addition, it has been reported that the degradation of tumor suppressor proteins p73 and p33 is regulated by ubiquitination. Recently, it has been shown that NQO1 can also inhibit p73 and p33 degradation in the presence of $\mathrm{NAD}(\mathrm{P}) \mathrm{H}$, and protects them from $20 \mathrm{~S}$ proteasomal degradation $(44,63)$. Furthermore, NQO1 appears to regulate the degradative fate of other proteins, such as p33ING1b and C/EBP $\alpha(47,48)$. These finding suggest that NQO1 plays an important role as a gatekeeper, in regulating the proteasomal degradation of specific proteins.

\section{REGULATION OF NQO1 GENE AND CANCER PREVENTION}

The use of dietary compounds or synthetic chemicals to decrease the incidence of cancer has been established half a century ago (8). Numerous studies demonstrated that chemopreventive agents are Nrf2 inducers (8). Thus, the use of che- mopreventive agents to induce the Nrf2/KEAP1/ARE signaling pathway, leading to the elevation of the expression of NQO1 gene, has emerged as a promising strategy for cancer prevention $(8,9)$. As the name of the pathway suggests, three major components are important to the transcription of the NQO1 gene: (i) ARE, DNA consensus sequences that are located in the promoter regions of the genes $(53,64)$; (ii) Nrf2, one of leucine zipper transcription factor, binds to the ARE, thereby signaling transcription of target genes (43); (iii) Kelch-like ECH-associated protein 1 (KEAP1) binds Nrf2 and promotes its ubiquitination and proteasomal degradation by Cul3-based ligase $(8,9)$.

\section{Regulation of NQO1 gene expression}

Analysis of the human, mouse and rat genes for NQO1 showed that NQO1 is located at $16 q 22.1$ on the human chromosome, and mouse chromosome $8(52,53,65,66)$. The NQO1 gene consists of five introns and six exons for an approximate length of $20 \mathrm{~kb}$ (53). There is considerable homology between the human and rat NQO1 coding sequences $(85 \%)(65)$. The first two amino acids and the first nucleotide of the third amino acid are encoded by Exon 1, while the remaining 272 amino acids are encoded by exons 2-6 (53). NQO1 is regulated by two distinct regulatory elements in the $5^{\prime}$ flanking region of the NQO1 gene that are the ARE, called the EpRE (electrophile response element), and the XRE (xenobiotic response element), called the AhRE, both under basal and during oxidative stress conditions $(2,53)$. A variety of antioxidants, $\mathrm{H}_{2} \mathrm{O}_{2}$, and tumor promoters increase AREmediated NQO1 expression $(53,64)$. Many transcription factors can recognize ARE, TMAnnRTGAYnnnGCRwww, in vitro, indicating that this is a composite regulatory DNA sequences (53). Because the AP-1 binding sequences, TGASTMAG, are similar to the ARE sequences, GTGACnnnGC, AP-1 and leucine zipper proteins (bZIP) including Nrf1, Nrf2 and Maf, participate in the induction of NQO1 gene $(53,67)$. A model of ARE-mediated regulation of hNQO1 is proposed by Wasserman and Fah1 (68). The ARE core sequence (RTGAYnnn) interacts with the bZIP transcription factors (Jun, Fos, Fra, Nrf, Maf, Raf and NF-E2) (53). It has been known that the Nrf2-KEAP1/ARE signaling pathway is the major regulator of cytoprotective responses to oxidative and electrophilic stresses (69). XRE-mediated gene expression involves the liganded aromatic hydrocarbon receptor (AHR). The XRE-mediated gene expression is increased by PAS (Per, Arnt, Sim) family of proteins (68). The AHR/Arnt dimer interacts with the DNA sequences, XRE (70). TCDD and polycyclic aromatic hydrocarbons induce NQO1 gene expression (71). However, one study 8 reported that, in mouse hepatoma cells, TCDD-induced human NQO1 was ARE- mediated and not dependent on XRE (72).

\section{Chemoprevention by upregulating NQO1}

Environmental carcinogens, including quinones, are first metabolically activated via the phase I enzymes such as cyto- 


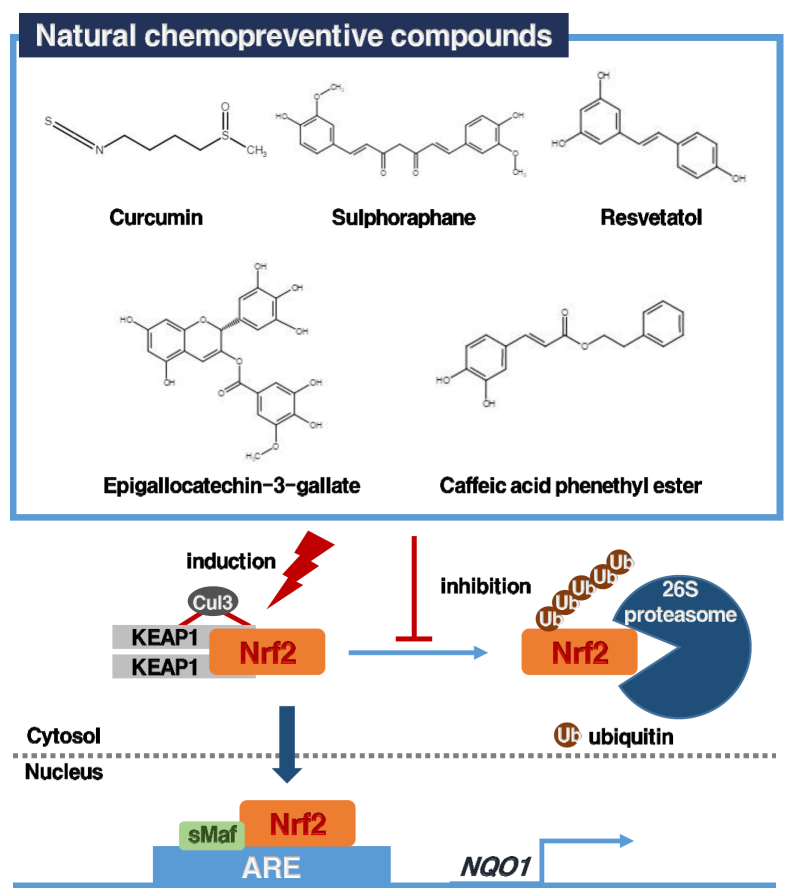

Fig. 2. Representative chemopreventive agents-induced activation of Nrf2/KEAP1/ARE signaling pathway and regulation of NQO1 gene.

chrome P450 into reactive intermediates (8). At the cellular level, there exist competing phase II enzymes (e.g. NQO1) which eliminate the reactive forms of carcinogens through biotransformation reactions, including quinone reduction, acetylation, sulfation and glutathione conjugation (8). Therefore, the use of dietary compounds or synthetic chemicals to shift the balance between phase I and II enzymes, is a promising strategy for cancer chemoprevention (73-75). The concept of chemoprevention strategy is closely correlated with Nrf2/KEAP1/ ARE signaling pathway-induced expression of NQO1 (8). Many studies reported that potent Nrf2 inducers can be obtained from plants including cruciferous vegetables (sulforaphane), a wide used spice (curcumin), green tea (epigallocatechin- 3-glaate), grapes (resveratrol), conifer trees (caffeic acid phenethyl ester) and Japanese horseradish (wasabi) (8). This chemopreventive natural compounds inducing Nrf2/NQO1 signaling pathway are continuously growing, and these have been reviewed comprehensively elsewhere (9). The signaling pathway is summarized in Fig. 2.

\section{NQO1 gene polymorphisms}

It has been known that NQO1 polymorphisms increase the susceptibility for developing cancer $(6,7,10-18)$. Two types of polymorphisms of the NQO1 gene have been reported in humans (43). The prominent one is a single nucleotide mutation, which is replacement of cytosine with thymidine $(609 \mathrm{C}>\mathrm{T})$, of the NQO1 gene $(13,43)$. This mutation produces a proline to serine substitutions at position 187 of the amino acid sequence of the NQO1 (6, 7, 10-18). Another mutation NQO $1 * 2$ protein is rapidly degraded by the proteasome (76). In addition, it has been reported that the null phenotype or deficiency for NQO1 increases the susceptibility to the neoplastic and toxic effects by benzene $(43,77)$. A large number of studies reported that the NQO1 polymorphisms correlate with the susceptibility for developing several types of cancer. However, the results showed inconsistency because of the small sample size in the majority of studies (16). In order to overcome the problem of low statistics, a few number of meta-analyses were performed in individual studies. However, these meta-analyses considered individual cancer sites separately (16). Therefore, a global meta-analysis to investigate the role of NQO1 polymorphisms should be conducted.

\section{THE POTENTIAL OF NQO1 FOR CANCER THERAPY}

Although absent or lowered NQO1 activity has been associated with the susceptibility for developing several types of human cancers as presented above $(6,7,10-18)$, the clinical significance of expression levels of NQO1 in human cancers has not been fully elucidated. In humans, NQO1 is overexpressed in a variety of solid tumors, including those of the adrenal gland, breast, colon, bladder, liver, ovary, cervix, pancreas lung, and thyroid $(6,22,23,78-80)$. In cancers, this feature has been exploited to activate anticancer drugs that are bioreductively activated by NQO1. In addition, there has been growing interest in the development of strategies to induce NQO1 activity in cancer cells for increasing the efficacy of bioreductive anticancer drugs.

\section{Bioreductive quinone substrates for NQO1}

Bioreductive anticancer drugs such as mitomycin $\mathrm{C}$ (MMC), $\beta$-lapachone and benzoquinone ansamycins, are activated by NQO1. MMC is a quinone containing antibiotic isolated from Streptomyces caespitosus. For more than 30 years, MMC has been used for the treatment of solid human tumors including breast, lung, pancreas and stomach (81). The mechanism of action of MMC is intracellular bioreductive activation which lead to DNA interstrand crosslinking (81). Since MMC is bioactivated by NQO1, the level of NQO1 is a good predictor of MMC sensitivity. In specifically hypoxic and acidic tumor microenvironments, other bioreductive enzyme can effectively activate MMC (82). Therefore, NQO1 expression level and NQO1 polymorphsim may not be important to determine the clinical response to MMC therapy (81). Another representative quinone, $\beta$-lapachone, is a naturally occurring ortho napthoquinone isolated from the bark of the lapachon tree (Tabeduia avellanedae) $(1,38,83,84)$. NQO1-induced activation of $\beta$-lapachone showed anti-trypanosomal, anti-fungal and anti- bacterial properties by production of hydrogen peroxide and superoxide with the simultaneous oxidation of reduced pyridine 
nucleotides (85). Early studies reported that $\beta$-lapachone could inhibit topoisomerase I, thereby inhibiting the repair of DNA in mammalian cells (81). $\beta$-lapachone (ARQ 501) is processed in the Phase I and II clinical trials for the cancer therapy (81). Finally, the benzoquinone ansamycins including geldanamycin (GA) and 17-AAG are a group of quinone. GA, which is isolated from Streptomyces hygroscopicus, has anticancer properties by inhibiting RNA and DNA replication (86). It has also been reported that GA could inhibit the activity of vSrc and inhibit the expression of the cMyc (81). In addition, GA targets the heat shock protein 90 (Hsp90) by inhibiting its ATPase activity (81). GA effectively inhibits Hsp90-mediated maturation of many oncogenic proteins such as HER2, Raf-1, $\mathrm{KIT}, \mathrm{BCR}-\mathrm{ABL}$ as well as steroid hormone receptors (81). Therefore, GA and its analogs such as 17-AAG and 17-DMAG have shown anticancer effect in various human cancers. NQO1 can convert 17-AAG to the hydroquinone of 17-AAG. The hydroquinone of 17-AAG, IPI504 (Retaspimycin), was developed as a more water-soluble alternative to 17-AAG. The IPI504 actively inhibits Hsp90 and shows markedly more potency than the parent quinone (87). In recent years, several new NQO1-dependent anticancer compounds have been developed, such as 2,5-diaziridinyl-3-3[hydroxymethyl]-6-methyl1,4-benzoquinone (RHI); 3-hydroxy-5-aziridinyl-1-methyl-2 [indol4,7- dione ]-prop-b-en-a- ol (EO9); and 3,4-dihydro-2, 2-dimethyl-2H-naphthol[1,2-b]pyran-5,6-dione ( $\beta$-lapachone) (83).

\section{Upregulation of NQO1 in cancer therapy with bioreductive anticancer drugs}

Cytotoxic quinone anticancer drugs may have the advantage of preferentially damaging the cancer cells when the cancer cells when the NQO1 enzyme is upregulated or overexpressed, relative to their action on cancer cells in normal conditions $(1,38,83,84)$. Recently, it has been shown that ionizing radiation at clinically relevant doses (e.g., 2 Gy) significantly upregulates the NQO1 level in cancer cells, and sensitizes the cells to $\beta$-lapachone $(38,39)$. When cells expressing NQO1 are treated with a combination of ionizing radiation and $\beta$-lapachone, positive feedback regulation between ROS and ERK leads to ER stress, inducing mitochondrial translocation of cleaved Bax and JNK activation. Subsequently, the decrease of mitochondrial membrane potential leads to translocation of AIF and apoptosis (84). We have also recently reported that hyperthermia (e.g. $41-42^{\circ} \mathrm{C}$ ) increases the enzymatic activity of NQO1 and Hsp70-mediated stabilization of NQO1, and sensitizes the cells to $\beta$-lapachone in vitro $(41,52$, 83). Heat shock elevates NQO1 expression by cis-acting elements such as ARE and XRE. The degradation of NQO1 protein in heat-treated cancer cells was slower than in untreated cells. After heating, the Hsp70 co-localized and co-precipitated with NQO1 in cancer cells, indicating the association of these two proteins in cancer cells (83). In addition, experimental mouse tumors or human tumor xenografts could be markedly sensitized to $\beta$-lapachone treatment by heating the tumors $24 \mathrm{~h}$ prior to $\beta$-lapachone treatment $(41,42)$. Furthermore, cisplatin significantly upregulates NQO1 in cancer cells, thereby markedly increasing the sensitivity of the cancer cells to $\beta$-lapachone in vitro as well as in vivo. These data suggested that local treatment of tumors with established cancer therapies such as hyperthermia or radiotherapy, which is summar-

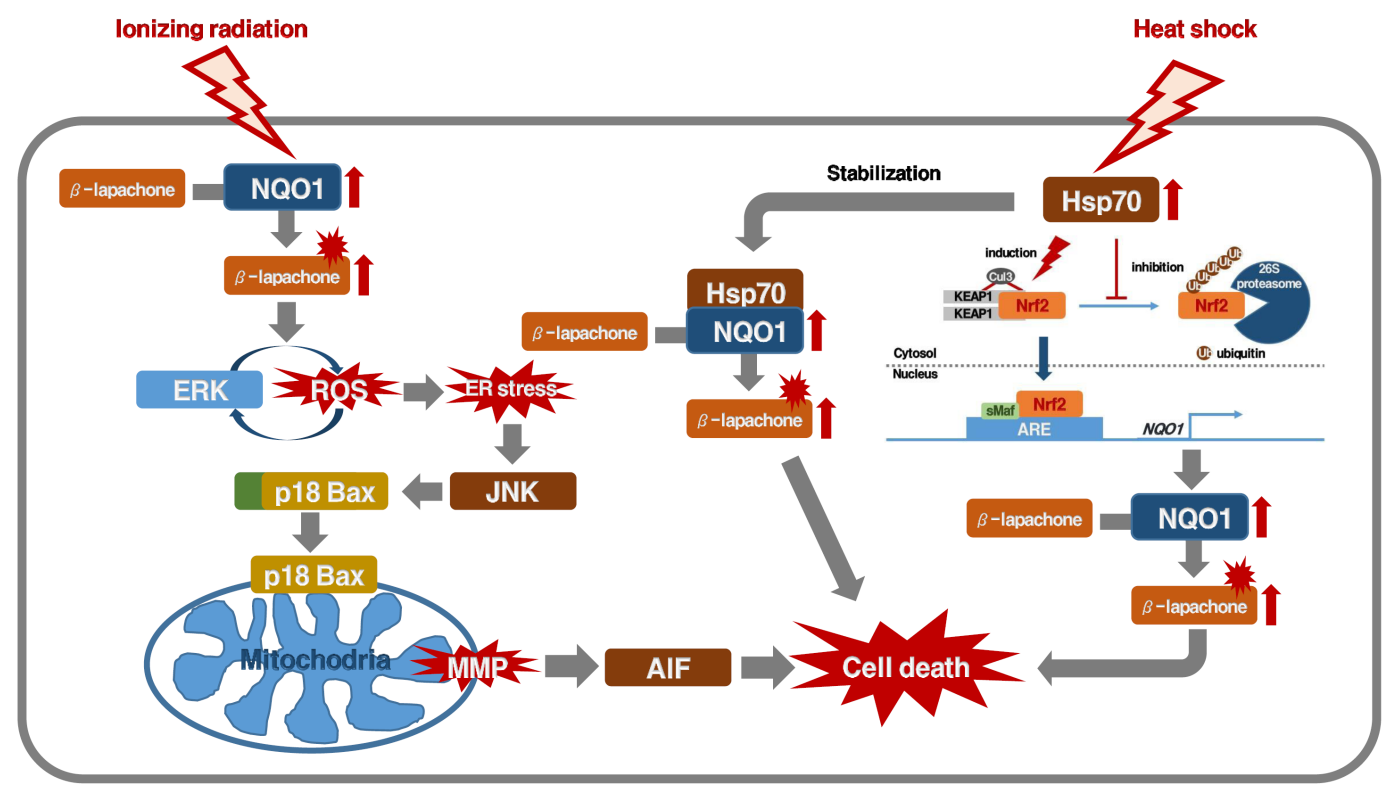

Fig. 3. Schematic model of how ionizing radiation or hyperthermia potentiates NQO1-dependent $\beta$-lapachone-induced cancer cell death. 
ized in Fig. 3, may upregulate NQO1 in the tumors and selectively sensitize the cancer cells to $\beta$-lapachone. Phase I and II clinical trials are conducted in progress to determine the feasibility of using $\beta$-lapachone alone or in combination with other anticancer drugs against human solid tumors (88). In order to improve the delivery and clinical efficacy of $\beta$-lapachone to tumors are being investigated (89-91).

\section{CONCLUSION}

There has been accumulating evidence exhibiting the versatile cytoprotective role of NQO1, in particular for cancer prevention and protection from oxidative stress-related diseases. A large number of studies reported the role of NQO1 polymorphisms in susceptibility for generation of several types of cancer. Importantly, certain compounds become cytotoxic due to reduction mediated by NQO1. Interestingly, NQO1 is overexpressed in providing an opportunity to preferentially damage cancers relative to normal tissues, using bioreductive anticancer drugs. Furthermore, there has been growing interest in the development of strategies to induce NQO1 activity in cancer cells for increasing efficacy of bioreductive anticancer drugs.

\section{ACKNOWLEDGEMENTS}

This research was supported by the National Research Foundation of Korea (NRF) grant funded by the Korea government (MSIP) (NRF-2013M2A2A7043703 and NRF-2014R1A5A2009392) and supported by INHA UNIVERSITY Research Grant.

\section{REPERENCES}

1. Terai K, Dong GZ, Oh ET et al (2009) Cisplatin enhances the anticancer effect of beta-lapachone by upregulating NQO1. Anticancer Drugs 20,901-909

2. Nioi $P$ and Hayes JD (2004) Contribution of $N A D(P) H$ : quinoneoxidoreductase 1 to protection against carcinogenesis, and regulation of its gene by the Nrf2 basic-region leucine zipper and the arylhydrocarbon receptor basic helix-loop-helix transcription factors. Mutat Res 555, 149-171

3. Kaspar JW and Jaiswal AK (2010) Antioxidant-induced phosphorylation of tyrosine 486 leads to rapid nuclear export of Bach 1 that allows Nrf2 to bind to the antitoxidant response element and activated defensive gene expression. J Biol Chem 285, 153-162

4. Venugopal R and Jaiswal AK (1998) Nrf2 and Nrf1 in association with Jun proteins regulate antioxidant response element-mediated expression and coordinated induction of genes encoding detoxifying enzymes. Oncogene 17, 3145-3156

5. Ernster L and Navazio F (1958) Soluble diaphorase in animal tissues. Acta Chem Scand 12, 595-602

6. Schlager JJ and Powis G (1990) Cytosilic NAD(P)H: (quinone-acceptor)oxidoreductase in human normal and tumor tissue: effects of cigarette smoking and alchol. Int J
Cancer 45, 403-409

7. Peng Q, Lu Y, Lao X et al (2014) The NQO1 Pro187Ser polymorphism and breast cancer susceptibility: evidence from an updated meta-analysis. Diagn Pathol doi: 10.1186/1746-1596-9-100

8. Cornblatt BS, Ye L, Dinkova-Kostova AT et al (2007) Preclinical and clinical evaluation of sulforaphane for chemoprevention in the breast. Carcinogenesis 28, 1485-1490

9. Surh YJ (2003) Cancer chemoprevention with dietary phytochemicals. Nat Rev Cancer 3, 768-780

10. Chen H, Lum A, Seifried A et al (1999) Association of the $\mathrm{NAD}(\mathrm{P}) \mathrm{H}$ :quinone oxidoreducatase $609 \mathrm{C} \rightarrow \mathrm{T}$ polymorphism with a decreased lung cancer risk. Cancer Res 59, 3045-3048

11. Lin $\mathrm{P}$, Wang $\mathrm{HJ}$, Lee $\mathrm{H}$ et al (1999) NAD(P)H:quinone oxidoreductase polymorphism and lung cancer in Taiwna. J Toxicol Environ Health A 58, 187-197

12. Lewis SJ, Cherry NM, Niven RM et al (2001) Polymorphisms in the $\mathrm{NAD}(\mathrm{P}) \mathrm{H}$ : quinone oxidoreductase gene and small cell lung cancer risk in a UK population. Lung Cancer 34, 177-183

13. Song SY, Jeong SY, Park HJ et al (2010) Clinical significance of NQO1 C609T polymorphisms after postoperative radiation therapy in completely resected nonsmall cell lung cancer. Lung Cancer 68, 278-282

14. Hamajima N, Matsuo $\mathrm{K}$, Iwata $\mathrm{H}$ et al (2002) NAD(P)H: quinone oxidoreductase 1 (NQO1) C609T polymorphism and the risk of eight cancers for Japanese. Int J Clin Oncol 7, 103-108

15. Menzel HJ, Sarmanova J, Soucek P et al (2004) Association of NQO1 polymorphsim with spontaneous breast cancer in two independent populations. Br J Cancer 90, 1989-1994

16. Lajin B and Alachkar A (2013) The NQO1 polymorphsim C609T (Pro187Ser) and cancer susceptibility: a comprehensive meta-analysis. Br J Cancer 109, 1325-1337

17. Fan $Y$, Hu D, Feng B and Wang W (2014) The NQO1 C609T polymorphism and hepatocellular carcinoma risk. Tumour Biol 35, 7343-7350

18. Cho CG, Lee SK, Nam SY et al (2006) Association of the GSTP1 and NQO1 polymorphsms and head and neck squamous cell carcinoma risk. J Korean Med Sci 21, 1075-1079

19. Zheng B, Wang Z and Chai R (2014) NQO1 C609T polymorphism and colorectal cancer susceptibility: a metaanalysis. Arch Med Sci 10, 651-660

20. Anwar A, Siegel D, Kepa JK et al (2002) Interaction of the molecular chaperone $\mathrm{Hsp} 70$ with human $\mathrm{NAD}(\mathrm{P}) \mathrm{H}$ : quinone oxidoreductase 1. J Biol Chem 277, 14060-14067

21. Traver RD, Horikoshi T, Danenberg KD et al (1992) $\mathrm{NAD}(\mathrm{P}) \mathrm{H}$ :quinone oxidoreductase gene expression in human colon carcinoma cells: characterization of a mutation which modulates DT-diaphorase activity and mitomycin sensitivity. Cancer Res 52, 797-802

22. Ma Y, Kong J, Yan G et al (2014) NQO1 overexpression is associated with poor prognosis in squamous cell carcinoma of the uterine cervix. BMC Cancer 14, 414. doi: 10.1186/1471-2407-14-414

23. Yang Y, Zhang Y, Wu Q et al (2014) Clinical implications of high NQO1 expression in breast cancers. J Exp Clin 
Cancer Res 33,14. doi: 10.1186/1756-9966-33-14

24. Cresteil T and Jaiswal AK (1991) High levels of expression of the $\mathrm{NAD}(\mathrm{P}) \mathrm{H}$ : quinone oxidoreductase (NQO1) gene in tumor cells compared to normal cells of the same origin. Biochem Pharmacol 42, 1021-1027

25. Siegel D, Franklin WA and Ross D (1998) Immunohistochemical detection of $\mathrm{NAD}(\mathrm{P}) \mathrm{H}$ : quinone oxidoreductase in human lung and lung tumors. Clin Cancer Res 4, 20652070

26. Beall HD, Murphy AM, Siegel D et al (1995) Nicotinamide adenine dinucleotide (phosphate): quinone oxidoreductase (DT-diaphorase) as a target for bioreductive antitumor quinones: quinone cytotoxicity and selectivity in human lung and breast cancer cell lines. Mol Pharmacol 48, 499-504

27. Siegel D, Gustafson DL, Dehn DL et al (2004) NAD(P)H: quinone oxidoreductase 1 : role as a superoxide scavenger. Mol Pharmacol 65, 1238-1247

28. Winski SL, Koutalos Y, Bentley DL et al (2002) Subcellular localization of $\mathrm{NAD}(\mathrm{P}) \mathrm{H}$ :quinone oxidoreducatase 1 in human cancer cells. Cancer Res 62, 1420-1424

29. Ross D, Kepa JK, Winski SL et al (2000) NAD(P)H:quinone oxidoreductase 1 (NQO1): chemoprotection, bioactivation, gene regulation and genetic polymorphisms. Chem Biol Interact 129, 77-97

30. Gibson NW, Hartley JA, Butler J et al (1992) Relationship between DT-diaphorase mediated metabolism of a series of aziridinyl benzoquinones and DNA damage and cytotoxicity. Mol Pharmacol 42, 531-536

31. Gustafson DL, Beall HD, Bolton EM et al (1996) Expression of human NQO1 (DT-diaphorase) in Chinese hamster ovary cells: effect on the toxicity of antitumor quinones. Mol Pharmacol 50, 728-735

32. Mikami K, Naito M, Tomida A et al (1996) DT-diaphorase as a critical determinant of sensitivity to mitomycin $C$ in human colon and gastric carcinoma cell lines. Cancer Res 56, 2823-2826

33. Ross D, Beall HD, Siegel D et al (1996) Enzymology of bioreductive drug activation. Br J Cancer 74 (suppl. XXVII), S1-S8

34. Kelland LR, Sharp SY, Rogers PM et al (1999) DT-Diaphorase expression and tumor cell sensitivity to 17-allylamino, 17-demethoxygeldanamycin, an inhibitor of heat shock protein 90. J Natl Cancer Inst 91, 1940-1949

35. Winski SL, Swann E, Hargreaves RH et al (2001) Relationship between $\mathrm{NAD}(\mathrm{P}) \mathrm{H}$ :quinone oxidoreductase 1 (NQO1) levels in a series of stably transfected cell lines and susceptibility to antitumor quinones. Biochem Pharmacol 61, 1509-1516

36. Loadman PM, Bibby MC, and Phillips RM (2002) Pharmacological approach towards the development of indole quinone bioreductive drugs used on the clinically inactive agent EO9. Br J Pharmacol 137, 701-709

37. Goetz MP, Toft D, Reid J et al (2005) Phase I trial of 17-allylamino-17-demethoxygeldanamycin in patients with advanced cancer. J Clin Oncol 23, 1078-1087

38. Choi EK, Terai K, Ji IM et al (2007) Upregulation of $\mathrm{NAD}(\mathrm{P}) \mathrm{H}$ :Quinone Oxidoreductase by radiation potentiates the effect of bioreductive $\beta$-lapachone on cancer cells. Neoplasia 9, 634-642
39. Park HJ, Ahn KJ, Ahn SD et al (2005) Susceptibility of cancer cells to beta-lapachone is enhanced by ionizing radiation. Int J Radiat Oncol Biol Phys 61, 212-219

40. Suzuki M, Amano M, Choi J et al (2006) Synergistic effects of radiation and beta-lapachone in DU-145 human prostate cancer cells in vitro. Radiat Res 165, 525-531

41. Park HJ, Choi EK, Choi J et al (2005) Heat-induced up-regulation of $\mathrm{NAD}(\mathrm{P}) \mathrm{H}$ : quinone oxidoreductase potentiates anticancer effects of beta-lapachone. Clin Cancer Res 11, 8866-8871

42. Song CW, Chae JJ, Choi EK et al (2008) Anti-cancer effect of bio-reductive drug beta-lapachon is enhanced by activating NQO1 with heat shock. Int J Hyperthermia 24, 161-169

43. Dinkova-Kostova A and Talalay $P(2010)$ NAD(P)H:quinone acceptor oxidoreducatase 1 (NQO1), a multifunctional antioxidant enzyme and exceptionally versatile cytoprotector. Arch Biochem Biophys 501, 116-123

44. Asher G, Tsvetkov P, Kahana C et al (2005) A mechanism of ubiquitin-independent proteasomal degradation of the tumor suppressors p53 and p73. Genes Dev 19, 316- 321

45. Asher G, Lotem J, Kama R et al (2001) NQO1 stabilizes p53 through a distinct pathway. Proc Natl Acad Sci U S A 99, 3099-3104

46. Anwar A, Dehn D, Siegel D et al (2003) Interaction of human $\mathrm{NAD}(\mathrm{P}) \mathrm{H}:$ Quinone Oxidoreductase 1 (NQO1) with the tumor suppressor protein p53 in cells and cell-free systems. J Biol Chem 278, 10368-10373

47. Garate M, Wong RPC, Campos El et al (2008) NAD(P)H quinone oxidoreductase 1 inhibits the proteasomal degradation of the tumour suppressor p33ING1b. EMBO Rep 9, 576-581

48. Patrick BA and Jaiswal AK (2012) Stress-induced NQO1 controls stability of $\mathrm{C} / \mathrm{EBP} \alpha$ against $20 \mathrm{~S}$ proteasomal degradation to regulate p63 expression with implications in protection against chemical-induced skin cancer. Oncogene 31, 4362-4371

49. Cuendet M, Oteham CP, Moon RC et al (2006) Quinone reductase induction as a biomarker for cancer chemoprevention. J Nat Prod 69, 460-463

50. Zhu $\mathrm{H}$ and $\mathrm{Li} \mathrm{Y}(2012) \mathrm{NAD}(\mathrm{P}) \mathrm{H}$ :quinone oxidoreductase 1 and its potential protective role in cardiovascular diseases and related conditions. Cardiovasc Toxicol 12, 39-45

51. Williams JB, Lu AYH, Cameron RG et al (1986) Rat liver $\mathrm{NAD}(\mathrm{P}) \mathrm{H}$ :quinone reductase. J Biol Chem 261, 5524-5528

52. Jaiswal AK, McBride OW, Adensik M et al (1988) Human dioxin-inducible cytosolic $\mathrm{NAD}(\mathrm{P}) \mathrm{H}$ :quinone oxidoreductase. J Biol Chem 263, 13572-13578

53. Ross D, Kepa JK, Winski SL et al (2000) NAD(P)H:quinone oxidoreductase 1 (NQO1): chemoprotection, bioactivation, gene regulation and genetic polymorphisms. Chem Biol Interact 129, 77-97

54. Atia A, Alrawaiq N and Abdullah A (2014) A review of $\mathrm{NAD}(\mathrm{P}) \mathrm{H}$ :quinone oxidoreductase 1 (NQO1); A multifunctional antioxidant enzyme. J Appl Pharm Sci 4, 118-122

55. Nebert DW, Roe AL, Vandale SE et al (2002) NAD(P)H: quinone oxidoreductase (NQO1) polymorphism, exposure to benzene, and predisposition to disease: a HuGE review. Genet Med 4, 62-70 
56. Lind C, Cadenas E, Hochstein P et al (1990) DT-diaphorase: purification properties and function. Methods Enzymol $186,287-301$

57. Wefers H and Sies H (1983) Hepatic low-level chemiluminescence during redox cycling of menadione and the menadione-glutathion conjugate: relation to glutathione and $\mathrm{NAD}(\mathrm{P}) \mathrm{H}$ :quinone reductase (DT-diaphorase) activity. Arch Biochem Biophys 224, 568-578

58. Li R, Bianchet MA, Talalay P and Amzel LM (1995) The three dimensional structure of $\mathrm{NAD}(\mathrm{P}) \mathrm{H}$ :quinone reductase, a flavoprotein involved in cancer chemoprotection and chemotherapy: mechanism of the two electron reduction. Proc Natl Acad Sci U S A 92, 8846-8850

59. Talalay $P$, and Dinkova-Kostova AT (2004) Role of nicotinamide quinone oxidoreductase 1 (NQO1) in protection against toxicity of electrophiles and reactive oxygen intermediates. Meth Enzymol 382, 355-364

60. Cenas N, Anusevicius Z, Nivinskas H et al (2004) Structure-activity relationships in two-electron reduction of quinones. Meth Enzymol 382, 258-277

61. Ross D (2004) Quinone reductases multitasking in the metabolic world. Drug Metabol Rev 36, 639-654

62. Siegel D and Ross D (2000) Immunodetection of $\mathrm{NAD}(\mathrm{P}) \mathrm{H}$ : quinone oxidoreductase 1 (NQO1) in human tissues. Free Radical Bio Med 29, 246-253

63. Asher G, Bercovich Z, Tsvetkov P et al (2005) 20S proteasomal degradation of ornithine decarboxylase is regulated by NQO1. Mol Cell 17, 645-655

64. Li Y and Jaiswal AK (1994) Human antioxidant response element mediated regulation of type $1 \mathrm{NAD}(\mathrm{P}) \mathrm{H}$ : quinone oxidoreductase gene expression. Eur J Biochem 226, 31-39

65. Vasiliou V, Theurer MJ, Puga A et al (1994) Mouse dioxin-inducible $\mathrm{NAD}(\mathrm{P}) \mathrm{H}$ : menadione oxidoreductase: $\mathrm{NMO} 1$ cDNA sequence and genetic differences in mRNA levels. Pharmacogenetics 4, 341-348

66. Robertson JA, Chen $\mathrm{H}$ and Nebert DW (1986) $\mathrm{NAD}(\mathrm{P}) \mathrm{H}$ : menadione oxidoreductase. Novel purification of enzyme, cDNA and complete amino acid sequence and gene regulation. J Biol Chem 261, 15794-15799

67. Itoh K, Chiba T, Takahashi $S$ et al (1997) An Nrf2:small Maf heterodimer mediates the induction of phase II detoxifying enzyme genes through antioxidant response elements. Biochem Biophys Res Commun 236, 313-322

68. Wasserman WW and Fahl WE (1997) Functional antioxidant responsive elements. Proc Natl Acad Sci U S A 94, 5361-5366

69. Li Y, Paonessa JD and Zhang Y (2012) Mechanism of chemical activation of Nrf2. PLoS One 7, e35122

70. Landers JP and Bunce NJ (1991) The Ah receptor and the mechanism of dioxin toxicity. Biochem I 276, 273-287

71. Kumaki K, Jensen NM, Shire JGM et al (1977) Genetic differences in induction of cytosol reduced-NAD(P): menadione oxidoreductase and microsomal aryl hydrocarbon hydroxylase in the mouse. J Biol Chem 252, 157-165

72. Radjendirane $\vee$ and Jaiswal (1999) Antioxidant response element-mediated 2,3,7,8-tetrachlorodibenzo-p-dioxin (TCDD) induction of human $\mathrm{NAD}(\mathrm{P}) \mathrm{H}$ :quinone oxidoreductase 1 gene expression. Biochem Pharmacol 58, 1649-1655

73. Sporn MB (1996) The war on cancer. Lancet 347,
1377-1381

74. Kensler TW (1997) Chemoprevention by inducers of carcinogen detoxication enzymes. Environ. Health Perspect 105, 965-970

75. Fahey JW, Talalay P (1999) Antioxidant functions of sulforaphane: a potent inducer of Phase II detoxication enzymes. Food Chem Toxicol 37, 973-979

76. Siegel D, Anwar A, Winski SL et al (2001) Rapid polyubiquitination and proteasomal degradation of a mutant from of $\mathrm{NAD}(\mathrm{P}) \mathrm{H}$ :quinone oxidoreductase 1. Mol Pharmacol 59, 263-268

77. Rothman N, Smith MT, Hayes RB et al (1997) Benzene poisoning, a risk factor for hematological malignancy, is associated with the NQO1 609C $\rightarrow$ T mutation and rapid fractional excretion of chlorzoxazone. Cancer Res 57, 2839-2842

78. Malkinson AM, Siegel D, Forrest DL et al (1992) Elevated DT-diaphorase activity and messenger RNA content in human non-small cell lung carcinoma: relationship to the response of lung tumor xenografts to mitomycin $\mathrm{Cl}$. Cancer Res 52, 4752-4757

79. Siegel D and Ross D (2000) Immunodetection ofNAD(P): quinone oxidoreductase 1 (NQO1) in human tissues. Free Rad Biol Med 29, 246-253

80. Cancer Genome Atlas Research Network (TCGA) (2012) Comprehensive genomic characterization define human colon and rectal cancer. Nature 487, 330-337

81. Siegel D, Yan C and Ross D (2012) NAD(P)H:quinone oxidoreductase 1 (NQO1) in the sensitivity and resistance to antitumor quinones. Biochem Pharmacol 83, 1033-1040

82. Begleiter A, Robotham E and Leith MK (1992) Role of $\mathrm{NAD}(\mathrm{P}) \mathrm{H}$ :(quinone acceptor) oxidoreductase (DT-diaphorase) in activation of mitomycin $\mathrm{C}$ under hypoxia. Mol Pharmacol 41, 677-682

83. Dong GZ, Youn H, Park MT et al (2009) Heat shock increases expression of $\mathrm{NAD}(\mathrm{P}) \mathrm{H}$ :quinone oxidoreductase (NQO1), mediator of beta-lapachone cytotoxicity, by increasing NQO1 gene activity and via Hsp70-mediated stabilization of NQO1 protein. Int J Hypethermia 25, 477-487

84. Park MT, Song MJ, Lee $\mathrm{H}$ et al (2011) $\beta$-lapachone significantly increases the effect of ionizing radiation to cause mitochondrial apoptosis activation in cancer cells. PLoS One 6, e25976

85. Boveris A, Docampo R, Turrens JF et al (1978) Effect of beta-lapachone on superoxide anion and hydrogen peroxide production in Trypanosoma cruzi. Biochem J 175, 431-439

86. Li LH, Clark TD, Cowie CH et al (1977) Effects of geldanamycin and its derivatives on RNA-directed DNA polymerase and infectivity of Rauscher leukemia virus. Cancer Treat Rep 61, 815-824

87. Guo W, Reigan P, Siegel D et al (2005) Formation of 17-allylamino-demethoxygeldanamycin (17-AAG) hydroquinone by $\mathrm{NAD}(\mathrm{P}) \mathrm{H}$ :-quinone oxidoreductase 1 : role of $17-A A G$ hydroquinone in heat shock protein 90 inhibition. Cancer Res 65, 10006-10015

88. Khong HT, Dreisbach L, Kindler HL et al (2007) A phase 2 study of ARQ 501 in combination with gemcitabine in adult patients with treatment naïve, unresectable pancre- 
atic adenocarcinoma. ASCO Annual Meeting Proceedings Part I. J Clin Oncol 25, 15017

89. Blanco E, Bey EA, Dong Y et al (2007) Beta-lapachonecontaining PEG-PLA polymer micelles as novel nanotherapeutics against NQO1-overexpressing tumor cells. J Control Release 122, 365-374
90. Dong Y, Chin SF, Blanco E et al (2009) Intratumoral delivery of beta-lapachone via polymer implants for prostate cancer therapy. Clin Cancer Res 15, 131-139

91. Park C, Youn H, Kim H et al (2009) Cyclodextrin-covered gold nanoparticles for targeted delivery of an anti-cancer drug. J Mater Chem 19, 2310-2315 